\section{SPINAL ANESTHESIA.}

THE visit of Prof. Thomas Jonnesco, of the University of Bucharest, to the Seamen's Hospital, Greenwich, has brought prominently before the public the method of producing local anæsthesia by the injection of anæsthetic solutions into the spinal canal.

Cocaine was introduced years ago as an anæsthetic fo local application; it was welcomed by the medical profession, and equally by patients, on account of its invaluable services in operative procedures upon the eye, the nose and the throat. By merely placing a drop or two of a solution of cocaine (or one of its salts) into the eye, or by painting a similar solution upon the mucous membrane of the nose or throat, it is possible to produce anæsthesia so complete as to enable surgical operations to be performed upon these parts without inflicting the least pain or discomfort upon the patients. Certain objections to the use of cocaine were not long, however, in showing themselves. Cocaine is a powerful alkaloid; and if the usual dose be exceeded, very grave results follow, a number of patients having actually died as the result of cocaine poisoning. Investigators were therefore led to search for other substances, either like cocaine occurring naturally, or prepared synthetically, which would possess the properties of cocaine while being less poisonous.

In this way a number of anæsthetic drugs has been introduced, including alypin, holocaine, eucaine (alpha and beta), scopolamine, novocaine, stovaine and tropacocaine. Of these the three latter have been chiefly employed in producing spinal anæsthesia. The method consists in injecting, by means of a syringe and needle, a quantity (usually about $I$ c.c.) of a solution of one of these substances into the spinal canal. The injection is made in the back, close to the middle line, the needle being inserted between two of the vertebræ. With regard to the details of the method, various procedures have been described, and no agreement has yet been reached as to which of these is to be considered the best. There is no doubt that modifications are desirable to suit particular requirements. Thus, many operators direct that the drug be dissolved in cerebro-spinal fluid or else in a saline solution having the same specific gravity and the same osmotic tension as the blood-serum. Others consider that the anæsthetic solution should be considerably denser or more viscous than the cerebrospinal fluid, and for this purpose recommend the addition of glucose or of gum-acacia to the solution. These thicker solutions tend to remain at the spot at which they are injected, while solutions in cerebrospinal fluid or in normal saline tend to spread up and down the spinal canal, and thus have a more widespread anæesthetic effect. It is usual to withdraw a few c.cm. of cerebro-spinal fluid from the spinal canal before injecting the anæsthetic fluid. There are two reasons for this-first, the surgeon is assured that he has actually introduced his needle into the spinal canal, and secondly he is certain to avoid increasing unduly the cerebro-spinal pressure when he introduces the anæsthetising fluid.

On introducing the fluid into a particular part of the spinal column, anæsthesia is produced of all parts of the body deriving their nerve supply from this part of the spinal cord, and all parts below. If the fluid be allowed to ascend the spinal canal (e.g. by raising the hips) the anæsthesia rises higher and higher as the anæsthetic fluid reaches the trunks of the nerves arising from the higher parts of the spinal cord. If the patient be placed on one side while the injection is being performed, the anæsthetic fluid can be made to enter one lateral half of the spinal canal, and in NO. 209I, VOL. 82] this way it is possible to limit the anæsthesia to one lateral half of the body.

The anæsthetic fluid can be allowed to ascend almost to the top of the thoracic spine without fear of untoward consequences. When it reaches the base of the neck, however, the phrenic nerve, concerned with the movements of respiration, becomes involved, and it was deemed impracticable to produce anæsthesia of the head and neck by the spinal method. Prof. Jonnesco, however, has shown that the addition of strychnine to the anæsthetic solution produces so powerful a stimulant effect upon the respiratory centre in the brain that it is possible to introduce an anæsthetic fluid into the upper part of the thoracic spine, and to allow the fluid to ascend the spinal canal in the neck so as to enable operations to be performed upon the neck and throat. But it is as yet too early to say whether this method may be considered a safe one.

Of the three drugs which are now chiefly used for the production of spinal anæsthesia, stovaine is found to produce the most deleterious effect upon the kidneys, acute nephritis having followed its injection in quite a number of cases. Novocaine and tropacocaine are less injurious in this way, while they are equally efficacious as anæsthetics. It thus appears likely that they will supplant stovaine in the near future, and, in fact, tropacocaine in a one per cent. solution is already being largely used for the purpose in this country, the usual dose injected being about I $\frac{1}{4}$ grain.

No doubt further experience will lead to modifications in the present method of performing spinal anæsthesia which will result in its widespread use, as there are a great many cases in which a local anæsthetic is far more advantageous to both patient and surgeon than a general anæsthetic.

A. C. J.

\section{THE CAUSES OF THE GERMINATIVE PROCESSES OF SEEDS.}

$\mathrm{NE}$ of the most remarkable phenomena of vegetable life is the occurrence in its cycle of a resting period of varying duration, a period during which the vital functions seem entirely suspended or dormant, and the condition of the organism is hardly distinguishable from death. This stage is most common in connection with the reproductive processes, and can be seen to belong to the constitution of both spores and seeds. The more highly differentiated the structure which shows it, the more prolonged, apparently, can be this resting period, but sooner or later it gives place to the resumption of growth and vital activities.

The interpretation of the occurrence of this phase is rather a matter of inference than proof; probably it was originally concerned in the protection of the reproductive structure from adverse conditions of the environment, for not only is the life rendered dormant, but the resting organ is for the most part protected by modification of its tegumentary covering. In this condition it is able also to bear the severance of its organic connection with its parent, and to subserve the purposes of dispersal. It may, indeed, have arisen with special reference to the latter process alone.

The resumption of the growth and development of the reproductive body after the period of rest may be explained in a similar manner by the reversal of the adverse conditions, these being for the most part secured when its dispersal has been effected.

These superficial considerations are found, however, on reflection, to have others underlying them. Is the resting period of any advantage to the living substance of the reproductive structure, whether spore 